\title{
A System-Level Modelling of Noise in Coupled Resonating MEMS sensors ${ }^{+}$
}

\author{
Vinayak Pachkawade \\ University of Liege, 4000 Liège, Belgium; vpachkawade@uliege.be \\ + Presented at the 1st International Electronic Conference-Futuristic Applications on Electronics,1-30 \\ November 2020
}

Received: 30 August 2020; Accepted: 27 September 2020; Published: 30 October 2020

\begin{abstract}
This paper presents realistic system-level modeling of effective noise sources in a coupled resonating mode-localized MEMS sensors. A governing set of differential equations are used to build a numerical model of a mechanical noise source in a coupled-resonator sensor and an effective thermo-mechanical noise is quantified through the simulation performed via SIMULINK. On a similar note, an effective noise that stems from the electronic readout used for the coupled resonating MEMS sensors is also quantified. Various noise sources in electronic readout are identified and the contribution of each is quantified. A comparison between an effective mechanical and electronic noise in a sensor system aids in identifying the dominant noise source in a sensor system. A method to optimize the system noise floor for an amplitude-based readout is presented. The proposed models present a variety of operating conditions, such as finite quality factor, varying coupled electrostatic spring strength, and operation with in-phase and out-of-phase mode. The proposed models aim to study the impact of fundamental noise processes that govern the ultimate resolution into a coupled resonating system used for various sensing applications.
\end{abstract}

Keywords: coupled resonators; sensor; mode-localization; mechanical noise; electronics noise; readout; system-level models; Simulink

\section{Introduction}

In a typical MEMS resonator system, electronic circuits (typically a current to voltage converter also called trans-impedance amplifier) is used for readout and processing of the electrical signal (output motional current from a resonator) provided by the sensing element. A total noise level of the system is therefore due to the combined effect of the mechanical-thermal noise in the mechanical domain, the electrical noise of the (resistive) mechanical sensing element [1] and the input referred noise of the readout circuits [1-4]. In a MEMS sensor system, noise from a readout circuit dominates the noise performance [5]. However, the details of the mechanical-thermal noise should also be considered relevant, and a noise source (mechanical or electronic) that determines the detection limit of the sensor should be analysed.

In this paper, an attempt is made to model and quantify an effective noise into the two coupled resonator (WCR) system used for sensing applications. Through mathematical analysis and simulations obtained via Simulink model, a source of dominant noise source is identified. Also, an effective noise floor in the design is quantified. Following this, the finest possible resolution for the design is proposed.

\section{Noise Floor in a Coupled Resonator Sensor}


In order to understand the impact of a system noise on the performance of a coupled resonator (CR) sensor, a noise source was added into the model. An output response was analysed to determine the ultimate detection limit, i.e., resolution of a CR sensor.

\subsection{Thermo-Mechanical Noise in CR Sensor Design}

In this section, a model to quantify a magnitude of a thermal-mechanical noise is presented. Small moving parts in MEMS are especially susceptible to mechanical noise resulting from molecular agitation [1]. In ultra-low level signal detection, mechanical-thermal noise plays an important role in setting up the effective noise floor of a sensor system, and, thus a minimum detection limit. In the context of a CR sensor, it has been concluded that it is the thermal-mechanical noise that governs the ultimate detection limit of a CR sensor [2]. In this paper, a mechanical-thermal noise source in 2 DoF $\mathrm{CR}$ sensor is modelled and its impact on the resolution (i.e. lowest possible detectable physical quantity) is quantified for the amplitude based output of the design.

Consider a schematic representation of a 2-DoF CR sensor system as shown in Figure 1a. It consists of effective mass, $M_{i}$, spring constant, $K_{i}$ and damping constant, $c_{i},(i=1,2)$. A coupling spring, $K_{c}$ and a damping constant, $c_{c}$ that may exists between the two resonators are also shown. Design conditions are: $M_{1}=M_{2}=M, K_{m 1}=K_{m 2}=K_{m}, c_{1}=c_{2}=c_{c}=c$ and $K_{c} \ll K_{m}$. In the design, it is stated that $X_{j i}$ is the amplitude variations of a $j^{\text {th }}$ resonator $(j=1,2)$ at the $i^{\text {th }}$ mode of a frequency response $(i=$ $1,2)$ due to the noise induced into the system. Therefore, an expression of the output/s (considering a system involving an intrinsic (mechanical) and extrinsic (electronic) noise sources) may be obtained as follows: i) a minimum resolvable amplitude ratio (AR) shift, $\left(\frac{X_{1}}{X_{2}}\right)_{\min _{j i}}=\left|\frac{R_{n}-R_{0}}{R_{0}}\right|_{\min _{j i}}$ and ii) a minimum resolvable amplitude shift, $(X)_{\min _{j i}}=\left|\frac{A_{n}-A_{0}}{A_{0}}\right|_{\min _{j i}}$. A power spectral density (PSD) of an amplitude displacement noise of $j^{\text {th }}$ resonator $(j=1,2)$ at the $i^{\text {th }}$ mode of the frequency response $(i=1$, 2) can be investigated (through the Simulink model). An effect of a mechanical noise was modelled by adding a force term into the governing set of equations of motion (for $2 \mathrm{DoF}$ ) as follows

$$
\begin{aligned}
& M \ddot{X}_{1}+\left(c+c_{c}\right) \dot{X}_{1}+\left(K_{m}+K_{c}\right) X_{1}-c \dot{X}_{2}-K_{c} X_{2}=F_{n o i s e_{\_} r m s} \\
& M \ddot{X}_{1}+\left(c+c_{c}\right) \dot{X}_{1}+\left(K_{m}+K_{c}\right) X_{1}-c \dot{X}_{2}-K_{c} X_{2}=F_{n o i s e_{2} r m s}
\end{aligned}
$$

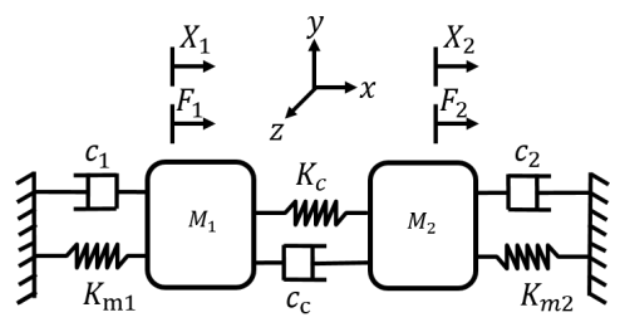

(a)

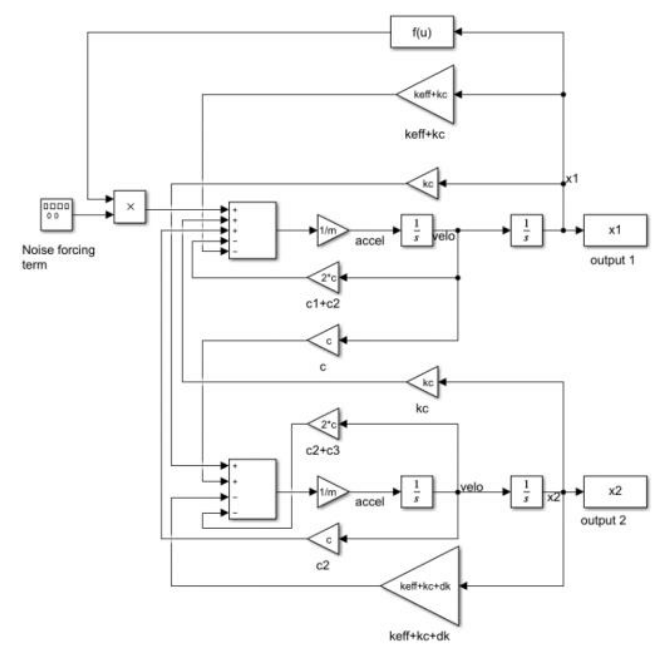

(b)

Figure 1. (a) Schematic representation of a 2-DoF CR sensor system. Proof mass, $M_{i}$, springs, $K_{i}$ and dampers, $c_{i}(i=1,2)$ are shown (b) Simulink block diagram representing system-level model. 
A spectral density of a noise forcing term is given as $F_{\text {noise_density }}=\sqrt{4 k_{B} T c} \mathrm{~N} / \mathrm{Hz}^{0.5}[1,5]$. Here, $k_{B}$ is the Boltzmann constant $\left(\approx 1.380 \times 10^{-23}\right.$ Joule/Kelvin), $T$ is the temperature (300 Kelvin) and $c$ is the damping coefficient $(c=0.0031 \mathrm{Ns} / \mathrm{m}$ in our case). As observed, the spectral density of a mechanical noise force depends on temperature and the magnitude of mechanical damping. A noise forcing term with the calculated average value, $5.136 \times 10^{-22} \mathrm{~N}^{2}$ was added as an excitation force in to the Simulink model shown in Figure 1b.

\section{Results}

A noise PSD (in $\mathrm{dB} / \mathrm{Hz}$ ) for displacements $X_{1}$ and $X_{2}$ was plotted as shown in Figure 2. Simulations were run for the varying strength of a coupling spring, $K_{c}$ between the two resonators in our design. Condition for the simulation are as follows: $c=0.0031 \mathrm{Ns} / \mathrm{m},(Q \approx 2547), \Delta k=0, \kappa=-0.0032$, excitation force applied to mass $M_{1} . \Delta k$ is the perturbation into the stiffness of one of the resonators in 2-DoF CR sensor system.

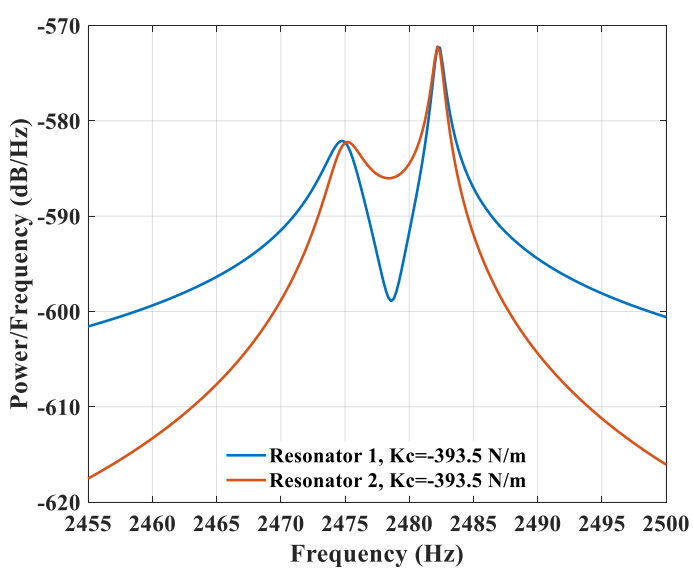

(a)

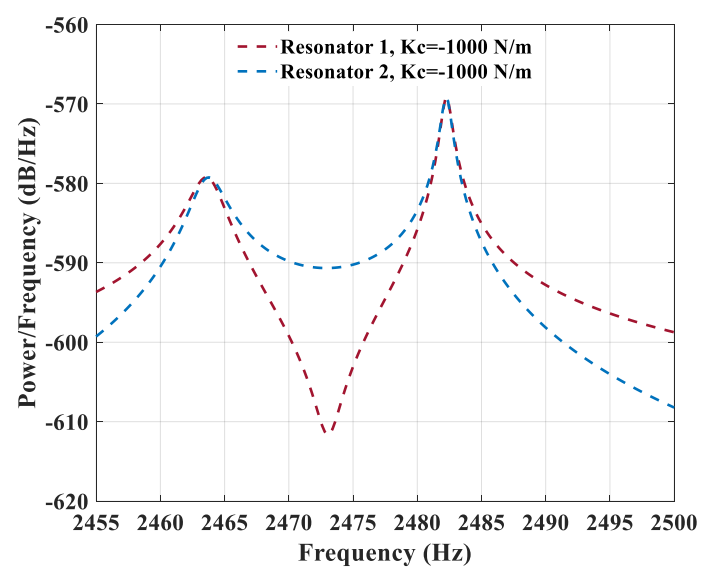

(b)

Figure 2. Simulated power spectrum density (PSD) of a displacement noise of resonator 1 and 2 subject to mechanical-thermal noise forcing term on resonator 1 . (a) $K_{c}=-393.5 \mathrm{~N} / \mathrm{m}$ and (b) $K_{c}=-1000$ $\mathrm{N} / \mathrm{m}$. Simulation conditions are as follows: $c=0.0031 \mathrm{Ns} / \mathrm{m},(Q \approx 2547), \Delta k=0$, excitation force applied to mass $M_{1}$. A normalized coupling factor, $\kappa$ is the given by $K c / K e f f . \Delta k$ is the induced perturbation into the stiffness of one of the resonator in 2-DoF CR sensor system.

A resultant displacement noise PSD (due to noise forcing term) is $-582.2 \mathrm{~dB} / \mathrm{Hz}$ (at mode 1 ) and $-572.2 \mathrm{~dB} / \mathrm{Hz}$ (at mode 2) (referring Figure 2a) which correspond to an equivalent magnitude $\left|X_{j}\right|$ of $7.76 \times 10^{-30} \mathrm{~m}^{2} / \mathrm{Hz}$ (at mode 1) and $2.45 \times 10^{-29} \mathrm{~m}^{2} / \mathrm{Hz}$ (at mode 2) respectively. Assuming a measurement bandwidth of $10 \mathrm{~Hz}$ around the resonant mode frequencies, $\omega_{i}(i=1,2)$, an average

Table 1. Table showing computation of the effective noise forcing term, displacement noise amplitudes and the resultant noise current due to the mechanical noise forcing term.

\begin{tabular}{|c|c|c|}
\hline Term & Value & Expression \\
\hline$X_{j 1_{-} a v g}$ & $\approx 7.762 \times 10^{-29} \mathrm{~m}^{2}$ & $X_{j i_{-} a v g}=\left|X_{j i}\right| \times d f^{1}$ \\
\hline$X_{j i \_ \text {rms }}$ & $\approx 8.81 \times 10^{-15} \mathrm{~m}$ & $X_{j 1_{1} \text { rms }}={\sqrt{X_{j 1_{\_} a v g}}}^{2}$ \\
\hline$i_{m o t} X_{j 1}$ & $\approx 4.29 \times 10^{-15} A_{r m s}$ & $i_{m o t X_{j i}}=\eta \omega_{i} X_{j i}^{3}$ \\
\hline$F_{\text {noise_}} P S D$ & $5.136 \times 10^{-23} N^{2} / H z$ & $\left(F_{\text {noise_density }}\right)^{2}=4 k_{B} T c^{*}$ \\
\hline$F_{\text {noise_avg }}$ & $5.136 \times 10^{-22} N^{2}$ & $F_{\text {noise_avg }}=\int_{0}^{10}\left(\sqrt{4 k_{B} T c}\right)^{2} d f$ \\
\hline$F_{n o i s e \_ \text {rms }}$ & $2.26 \times 10^{-11} N$ & $F_{\text {noise_rms }}=\sqrt{F_{\text {noise_avg }}} *$ \\
\hline
\end{tabular}


${ }^{1}$ A best-case estimate for average noise power for mode 1. ${ }^{2}$ An effective $r m s$ value of a mechanicalthermal noise for mode $1 .{ }^{3}$ An effective noise current due to the mechanical noise forcing term. ${ }^{\text {Power }}$ spectral density (PSD) of mechanical noise force generator. ${ }^{* *}$ Average (mean square) value of a mechanical thermal noise force generator, assumed measurement bandwidth is $10 \mathrm{~Hz} .{ }^{* * *} \mathrm{~A}$ effective rms amplitude of mechanical noise forcing term (mean square) value of a mechanical thermal noise of $j^{\text {th }}$ resonator at the $i^{\text {th }}$ mode $(j, i=1,2)$ will be $X_{j i_{a} a v g}=\left|X_{j i}\right| \times d f$. Refer Table 1 for the computations.

As observed from Figure 2, noise magnitude is lower for mode 1.

A theoretical estimation of effective noise current for our design (due to intrinsic mechanicalthermal noise) is given as $i_{\text {mot }_{X i}}=\eta \omega_{i} X_{j i}$, where, $\eta \omega_{i}$, and $X_{j i}$ are transduction factor, angular frequency and the maximum displacement amplitude of the $j^{\text {th }}$ resonator $(j=1,2)$ at the $i^{\text {th }}$ mode of the frequency response $(i=1,2)$, respectively [6]. By determining the $X_{j i}$, the effective mechanicalthermal noise current (i.e., corresponding variations in the motional currents, $i_{\text {mot } X_{j i}}$ due to noise forcing term) was quantified (refer Table 1). Since, a thermal noise amplitude of the mode 1 is relatively lower (referring Figure 2), the computation for the best case is given.

\subsection{Modelling the Noise from the Electronic Readout}

Figure 3a represents the schematic representation of transimpedance amplifier that may be realized using OPA 381 [7] integrated circuit (IC) as an interface circuit to the CR design. Two such ICs may be deployed for motional current pick-up into each output channel of the CR output. Figure $3 \mathrm{~b}$ is the equivalent noise model for the interface circuit used (transimpedance amplifier). A noise contribution from each of these sources are estimated by superposition and nodal analysis.

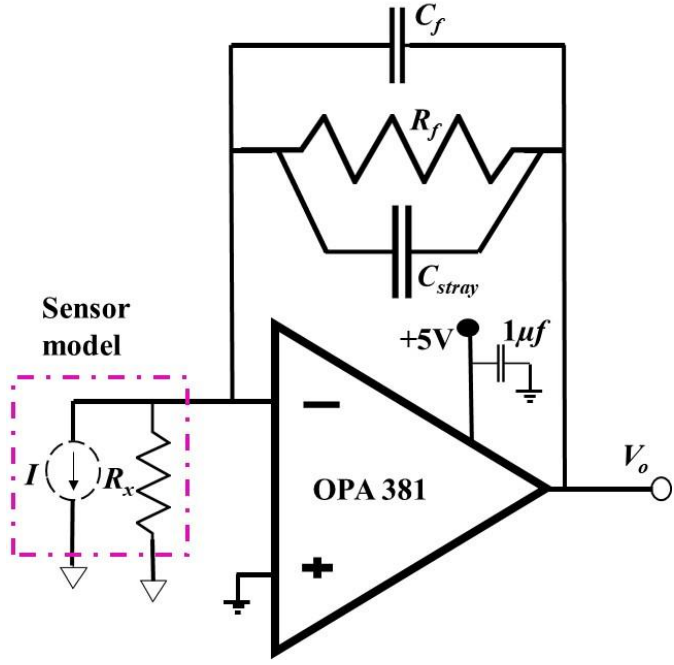

(a)

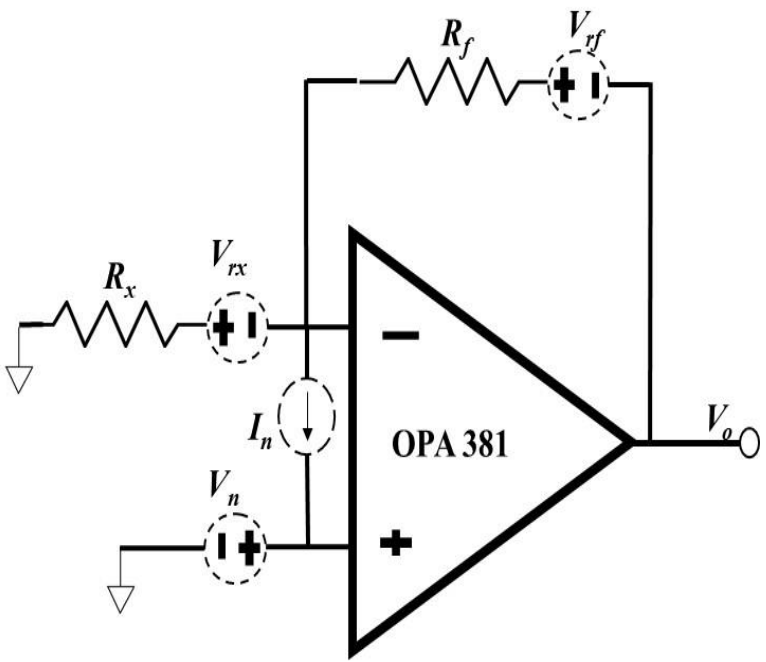

(b)

Figure 3. (a) OPA 381 interface for a 2-DoF CR sensor design (b) equivalent noise circuit to evaluate circuit dominant noise.

An amplifier (OPA 381) [7] used in the analysis has an input current noise density, $i_{n} \approx 20$ $\mathrm{fA} / \mathrm{Hz}^{0.5}$, and an input voltage noise density, $v_{n} \approx 70 \mathrm{nV} / \mathrm{Hz}^{0.5}$. In CR sensor system, in the presence of an applied ac signal $v_{a c}$ an impact of noise on the motional current output $i_{\text {mot }_{i j}}$ of $j^{\text {th }}$ resonator $(j=1$, 2 ) at the $i^{\text {th }}$ mode of a response $(i=1,2)$ can be evaluated (refer Figure $\left.1 \mathrm{~b}\right)$. This ac voltage signal together with the DC voltage, $V_{d c}$ exerts a net electrostatic actuation force at the resonant frequency. In order to evaluate the impact of the electronic preamplifier (OPA 381) noise on the minimum shifts into the amplitude based output/s (and thus quantify measurand, $\Delta k$ or $\delta_{k_{\min }}$ (normalized)), all noise 
components were re-expressed as an equivalent currents as given in Table 2. In Table 2, $i_{j}^{\text {nose }}$ (rms) is the noise current from the $j^{\text {th }}$ resonator at the $i^{\text {th }}$ mode of response. $R_{x}$ is the motional resistance, $R_{x}=\frac{d^{4} \sqrt{k_{e f f} M}}{V_{d c}^{2} \varepsilon^{2} A^{2} Q}$ [8] ( $R_{x}=4 \mathrm{M} \Omega$ for a $Q \approx 2547$ with other parameters being constant, in our case), $R_{f}$ is the effective value of a feedback resistor used in the preamplifier $(1 \mathrm{M} \Omega)$. The term $k_{B}$ is the Boltzmann constant $\left(\approx 1.380 \times 10^{-23}\right.$ Joule/Kelvin) and $T$ is the temperature (300 Kelvin). Term $B$ is the integration bandwidth of $10 \mathrm{~Hz}$ around the resonator's mode frequencies, $f_{-} i p$ and $f_{-o p}$ ) taken for computations for optimum noise estimation. From Table 2, an effective theoretical noise floor (i.e., input-referred electronic noise current, $i_{j}^{\text {noise }}$ due to electronic readout) of our design is $\approx 1.56 \times 10^{-13} A_{\text {rms. }}$. A total noise (mechanical resonator + electronics) in our design is then estimated by vector sum of the uncorrelated noise sources i.e. $I_{t^{2}}=I_{1}{ }^{2}+I_{2}{ }^{2}$, where, $I_{1}$ is effective mechanical noise current and $I_{2}$ is effective electronic readout noise current. $I_{t}$ is calculated to be $\approx 1.56 \times 10^{-13}$ Arms.

Table 2. Table showing contribution of the noise current due to the several noise terms used in the electronic readout for the mechanical CR sensor.

\begin{tabular}{|c|c|c|}
\hline Noise Sources & $\begin{array}{c}\text { Effective Noise } \\
\text { Current }\left(A_{r m s}\right)\end{array}$ & Expression \\
\hline $\begin{array}{c}\text { Mechanical thermal- } \\
\text { noise }^{1}\end{array}$ & $4.9 \times 10^{-15}$ & $i_{X_{j i}}=\eta \omega_{i} X_{j i}$ \\
\hline Feedback resistance & $4.06 \times 10^{-13}$ & $\sqrt{\frac{4 k_{B} T \times B}{R_{f}}}$ \\
\hline $\begin{array}{l}\text { Input voltage noise of a } \\
\text { pre-amplifier }\end{array}$ & $4.34 \times 10^{-13}$ & $\sqrt{v_{n}^{2}\left(1+\frac{R_{x}}{R_{f}}\right)^{2} / R_{x}^{2}} \times \sqrt{B} \times 1.57$ \\
\hline $\begin{array}{c}\text { Input current noise of a } \\
\text { pre-amplifier }{ }^{2}\end{array}$ & $9.92 \times 10^{-14}$ & $\sqrt{i_{n}^{2}} \times \sqrt{B} \times 1.57$ \\
\hline $\begin{array}{l}\text { input-referred electronic } \\
\text { noise current, } i_{j}^{\text {noise }}\end{array}$ & $1.56 \times 10^{-13} A_{r m s}$ & $i_{j}^{n \text { noise }}=\sqrt{\left(i_{j}^{\text {noise }}\right)^{2}}=\sqrt{\left[i_{n}^{2}+v_{n}^{2}\left(1+\frac{R_{x}}{R_{f}}\right)^{2} / R_{x}^{2}+\frac{4 k_{B} T}{R_{f}}\right.}$ \\
\hline $\begin{array}{c}\text { Total system noise } \\
\text { (mechanical + electronic) }\end{array}$ & $\approx 1.56 \times 10^{-13}$ Arms & $I_{\mathrm{t}^{2}}=I_{1}{ }^{2}+I_{2}{ }^{2}$ \\
\hline
\end{tabular}

${ }^{1}$ best-case calculation for the out-of-phase mode as it shows the lower noise amplitude. ${ }^{2}$ factor 1.57 is the roll-off rate of a filter (1-pole) [9].

An electrostatic forcing term was applied to the Simulink model shown in Figure 1b. An effective system noise was added to the Simulink model. Other model conditions are same as given in Figure 2. As a result, following computations are obtained (refer Table 3). Calculated values for motional current amplitudes are in agreement with the simulated values.

Table 3. Computations and simulated results in response to the electrostatic forcing term used in the Simulink model. A noise due to mechanical and electronic terms were taken into account to simulate for the output response.

\begin{tabular}{|c|c|c|c|}
\hline Term & Value (mode 1) & Value (mode 2) & Expression \\
\hline $\begin{array}{l}\text { maximum displacement }{ }^{1} \text {, } \\
\qquad\left|x_{j i}\right|\end{array}$ & $x_{j 1} \approx 0.419 \mu \mathrm{m}$ & $x_{j 2} \approx 0.836 \mu \mathrm{m}$ & N/A \\
\hline $\begin{array}{l}\text { motional current } \\
\text { amplitudes }^{2}, i_{\text {mot }_{j i}}\end{array}$ & $\approx 192 n A$ & $\approx 384 n A$ & $\begin{aligned} i_{m o t_{j 1}} & \approx \eta \times 2 \pi f_{-o p} \times x_{j 1} \\
i_{m o t_{j 2}} & \approx \eta \times 2 \pi f_{-i p} \times x_{j 2}\end{aligned}$ \\
\hline $\begin{array}{l}\text { Effective output voltage }{ }^{3}, \\
\qquad V_{\text {out }_{j i}}\end{array}$ & $=0.135 V_{r m s}$ & $=0.271 V_{r m s}$ & $\begin{array}{l}V_{\text {out }_{j 1}}=i_{\text {mot }_{j 1}} \times R_{f} \approx 0.192 V_{\max }, \\
V_{\text {out }_{j 2}}=i_{\text {mot }} \times R_{f 2} \approx 0.384 V_{\max }\end{array}$ \\
\hline
\end{tabular}


output referred noise voltage $^{2}, V_{j i}^{\text {noise }}$

lowest possible resolvable mode amplitude shift,

$$
\approx \frac{V_{j i}^{\text {noise }}}{V_{\text {out }_{j i}}}
$$

minimum resolvable shift in the amplitude ratio readout, $A R_{i}$ $\approx 156 \times 10^{-9} V_{r m s}$

N/A

$$
\begin{array}{cc}
\frac{156 \times 10^{-9}}{0.135} \approx 1.15 \times 10^{-6} \\
\begin{array}{c}
\text { (dimensionless) } \\
0.271
\end{array} \quad \text { (dimensionless) }
\end{array}
$$

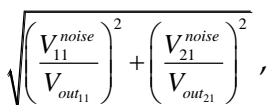

(dimensionless) $\approx 3.89 \times 10^{-1}$

(dimensionless)

$$
i_{j 1}^{\text {noise }} \times R_{f}
$$

$$
\frac{V_{j 1}^{\text {noise }}}{V_{\text {out }_{j 1}}}, \frac{V_{j 2}^{\text {noise }}}{V_{\text {out }_{j 2}}}
$$

${ }^{1}$ Derived from the Simulink. ${ }^{2}$ Theory. ${ }^{3}$ amplification factor, $R_{f}=1 \mathrm{M} \Omega$, as a feedback resistor in preamplifier OPA 381.

\subsection{Resolving the Lowest Possible Shifts (i.e., Resolution)}

The minimum resolvable shift in the voltage amplitudes of our 2-DoF CR sensor were derived as $\approx \frac{V_{j i}^{\text {noise }}}{V_{\text {out }_{j i}}}[6]$. Here, $V_{j i}^{\text {noise }}$ is the output refereed noise voltage of the $j^{\text {th }}$ coupled resonator at the $i^{\text {th }}$ mode. $V_{\text {out }_{j i}}$ is the noiseless deterministic output voltages (determined as $V_{\text {out }_{j i}}=i_{\text {mot }_{j i}} \times R_{f}$, where, $\left.i_{\text {mot }_{j} i}=\eta \omega_{i} x_{j i}\right)$. Therefore, for the $j^{\text {th }}$ resonator lowest possible resolvable mode amplitude shift, $\frac{V_{j 1}^{\text {noise }}}{V_{\text {out }_{j 1}}}$ is $\approx \frac{156 \times 10^{-9}}{0.135} \approx 1.15 \times 10^{-6}$, (dimensionless) for mode 1 , and $\frac{V_{j 2}^{\text {noise }}}{V_{\text {out }_{j 2}}}$ is $\approx \frac{156 \times 10^{-9}}{0.271} \approx 5.756 \times 10^{-7}$ (dimensionless) for mode 2. Similarly, minimum resolvable shift in the amplitude ratio readout, $A R_{i}$ at the $i^{\text {th }}$ mode $(i=1,2)$ is $\sqrt{\left(\frac{V_{11}^{\text {noise }}}{V_{\text {out }_{11}}}\right)^{2}+\left(\frac{V_{21}^{\text {noise }}}{V_{\text {out }_{21}}}\right)^{2}} \approx 1.481 \times 10^{-6}$ (dimensionless) for mode 1 and $\sqrt{\left(\frac{V_{12}^{\text {noise }}}{V_{\text {out }_{12}}}\right)^{2}+\left(\frac{V_{22}^{\text {noise }}}{V_{\text {out }_{22}}}\right)^{2}} \approx 3.89 \times 10^{-7}$ (dimensionless) for mode 2. Since rms amplitudes of output voltage (essentially a motional current) at the mode 1 for $j^{\text {th }}$ resonator is relatively closer (worst-case signal-to-noise ( $\mathrm{S} / \mathrm{N}$ ) ratio) to the rms noise amplitude, it gives the possibility to determine the worstcase lowest possible shift (thus resolution) in the design. The effective resolution (theoretical) of our design for AR based readout is $\approx 3.89 \times 10^{-7}$ which may be compared to the theoretically calculated resolution $\left(\approx 6 \times 10^{-3}\right)$ in MEMS coupled resonator design in [6].

\subsection{Influence of Coupling Strength on the Effective Noise Floor}

This section shows if electrostatic spring strength, $K_{c}$, coupling the two resonators influence the effective noise floor. Figure 4 shows a simulated frequency spectrum of the $j^{\text {th }}$ resonator $(j=1,2)$ resonators for two variants of coupling spring, $K_{c}$. As observed, with the given set of operating conditions, output signal from resonator 2 offers relatively lower noise floor as compared to resonator 1 and is independent of the coupling strength (refer to Figure $4 a, b)$. In addition, it is useful to utilize output of $j^{\text {th }}$ resonator at mode 2 as it offers a higher amplitude and thus maximum shifts (sensitivity). From Figure 4 , it is inferred that smaller coupling strength, $K_{c}$ leads to reduced noise floor (about -12 $\mathrm{dB}$ gain in the noise floor thus enhancing the ultimate detection limit of the measurand) for the resonating output of the resonator 2 . 


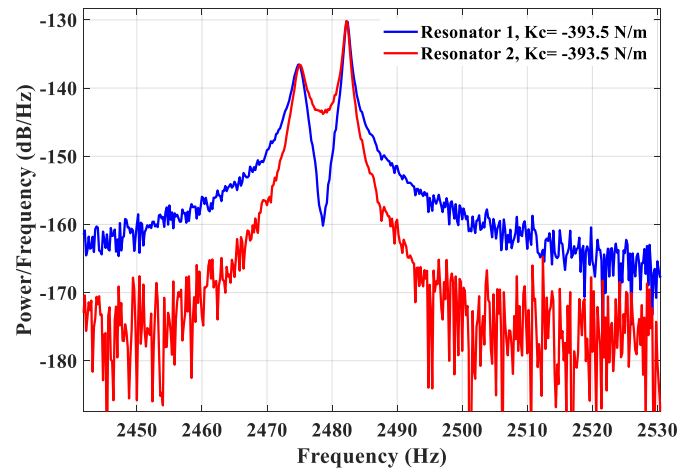

(a)

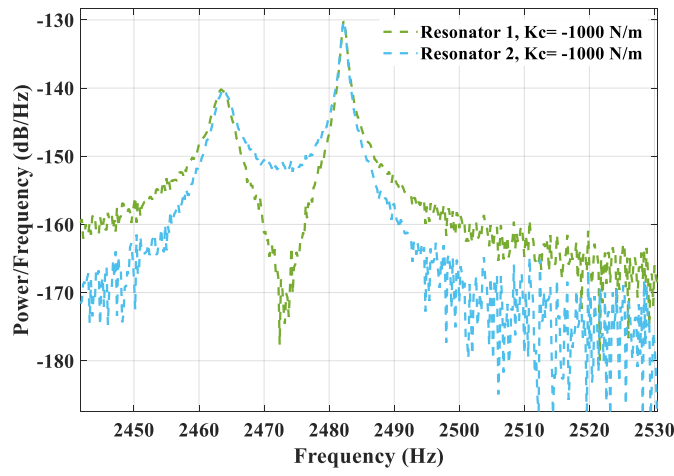

(b)

Figure 4. Simulated power spectrum density (PSD) of the motional current output signal in a two weakly coupled resonator for the following operating condition, $Q=2547, \delta_{k}=0, F=1 \mathrm{~N}$

\section{Discussion and Conclusion}

This work attempted to model and quantify the noise in the design of a 2-DoF MEMS CR sensor. An expected variation in the sensor output arising from the intrinsic (mechanical) and extrinsic (electronic) noise sources were individually evaluated via theory and system-level simulations. From Table 2, it is seen that though mechanical-thermal noise sets the ultimate noise floor of in this design, it is an electronic noise that dominates the overall performance (electronics noise exceeds mechanical noise by two orders of magnitude). Through quantifying the system noise in the system, a systemlevel model aids to simulate the sensor output response. And, the ultimate detection limit in the 2DoF CR sensor is shown to be determined. A method to optimize the system noise/improve detection limit is also shown (via tuning the coupling spring).

\section{References}

1. Gabrielson, T.B. Mechanical-Thermal Noise in Micromachined Acoustic and Vibration Sensors, IEEE Trans. Electron. Devices, 1993. doi:10.1109/16.210197.

2. Juillard, J.; Prache, P.; Ferreira, P.M.; Barniol, N. Ultimate limits of differential resonant MEMS sensors based on two coupled linear resonators, IEEE Trans. Ultrason. Ferroelectr. Freq. Control, 2018. doi:10.1109/TUFFC.2018.2869415.

3. Juillard, J.; Prache, P.; Ferreira, P.M.; Barniol, N. Impact of output metric on the resolution of mode-localized MEMS resonant sensors, 2017 Jt. Conf. Eur. Freq. Time Forum IEEE Int. Freq. Control Symp. EFTF/IFC, 2017. doi:10.1109/FCS.2017.8088943.

4. Pandit, M.; Zhao, C.; Sobreviela, G.; Mustafazade, A.; Du, S.; Zou, X.; Seshia, A.A. Closed-Loop Characterization of Noise and Stability in a Mode-Localized Resonant MEMS Sensor, IEEE Trans. Ultrason. Ferroelectr. Freq. Control, 2019. doi:10.1109/TUFFC.2018.2878241.

5. Djurić, Z. Mechanisms of noise sources in microelectromechanical systems, Microelectron. Reliab., 2002. doi:10.1016/s0026-2714(00)00004-4.

6. Thiruvenkatanathan, P.; Woodhouse, J.; Yan, J.; Seshia, A.A. Limits to mode-localized sensing using microand nanomechanical resonator arrays, J. Appl. Phys., 2011. doi:10.1063/1.3590143.

7. Precision, Low Power, $18 \mathrm{MHz}$ Transimpedance Amplifier, n.d. Available online: www.ti.com (accessed on 23 June 2019).

8. Hung, L.; Nguyen, C. High-Q Low-Impedance MEMS Resonators, UC Berkeley Electron. Theses Diss., 2012. Precision, Low Power, $18 \mathrm{MHz}$ Transimpedance Amplifier, n.d. Available online: www.ti.com (accessed on 23 June 2019).

9. TI Precision Labs-Op Amps: Noise 1 । TI.com Video, (n.d.). Available online: https://training.ti.com/tiprecision-labs-op-amps-noise-1 (accessed on 23 June 2019). 
(C) 2020 by the authors. Submitted for possible open access publication under the terms and conditions of the Creative Commons Attribution (CC BY) license (http://creativecommons.org/licenses/by/4.0/). 